\title{
In-Situ Liquid Phase Electron Microscopy of Beam-Sensitive Materials
}

$\underline{\text { Hanglong Wu}}^{1,3}$, Alessandro Ianiro ${ }^{2,3}$, Mark M. J. van Rijt ${ }^{1,3}$, Arthur D. A. Keizer ${ }^{1,3}$, A. Catarina C. Esteves $^{2,3}$, Remco Tuinier ${ }^{2,3}$, Heiner Friedrich ${ }^{1,3}$, Nico. A.J.M. Sommerdijk ${ }^{1,3}$ and Joseph P. Patterson $^{1,3}$

${ }^{1 .}$ Laboratory of Materials and Interface Chemistry \& Center of Multiscale Electron Microscopy, Department of Chemical Engineering and Chemistry, Eindhoven University of Technology, PO box 513, Eindhoven, The Netherlands.

2. Laboratory of Physical Chemistry, Department of Chemical Engineering and Chemistry, Eindhoven University of Technology, Eindhoven, The Netherlands.

3. Institute for Complex Molecular Systems, Eindhoven University, Eindhoven, The Netherlands.

Recent advances [1-4] in liquid phase electron microscopy (LP-EM) have provided unique capabilities for directly observing nanoscale dynamics in liquid with a unique combination of spatial and temporal resolution [5-6]. Of particular interests in this field is that to image beam-sensitive materials in aqueous solutions, which still remains challenging due to the low contrast and high beam sensitivity.

To minimize the $\mathrm{e}^{-}$beam driven radiolysis thus allowing for the videographic observation of nonradiolytic processes, low dose imaging should be applied [7]. This inherently decreases the signal-tonoise ratio (SNR) and limits the achievable spatial resolution. Therefore, it is of key importance to efficiently use the electron dose supplied to the materials and to extract the maximum information from the relatively high-noise data, while achieving an accurate interpretation.

Here we will discuss our efforts to perform quantitative image processing and analysis to extract dynamical information from $7 \mathrm{~nm} /$ pixel movie data recorded from low dose LP-EM imaging. The movie shows a block polymer self-assembly process (poly(caprolactone)- $b$-poly(ethylene oxide) PCL-b-PEO) initiated by a acetone $\rightarrow$ water solvent switch in liquid cell holder. To validate our observations, we compare our results with those collected from standard a low dose imaging technique, Cryo-TEM. Self-consistent field simulations have also been conducted to obtain a molecular understanding of this macromolecular self-assembly process. Our findings from data analysis and simulations enable us to offer a unifying description of the two well-established vesicle formation mechanisms. To better understand the beam effects in liquid, we investigate a second system where we vary the dose over 5 order of magnitude during imaging. We show that despite the dramatic decrease in dose we can still gain knowledge of the process captured under extremely low dose conditions.

\section{References:}

[1] N De Jonge and FM Ross, Nature Nanotechnology 6 (2016), p. 695.

[2] JJ De Yoreo and NAJM Sommerdijk, Nature Reviews Materials 1 (2016), p. 16035.

[3] FM Ross, Science 350 (2015), p. 6267.

[4] JM Yuk et al, Science 336 (2012), p. 61.

[5] PJ Smeets et al, Nature Materials 14 (2015), p. 394.

[6] JP Patterson et al, Journal of the American Chemical Society 137 (2015), p. 7322.

[7] LR Parent et al, Accounts of Chemical Research 51 (2018), p. 3. 


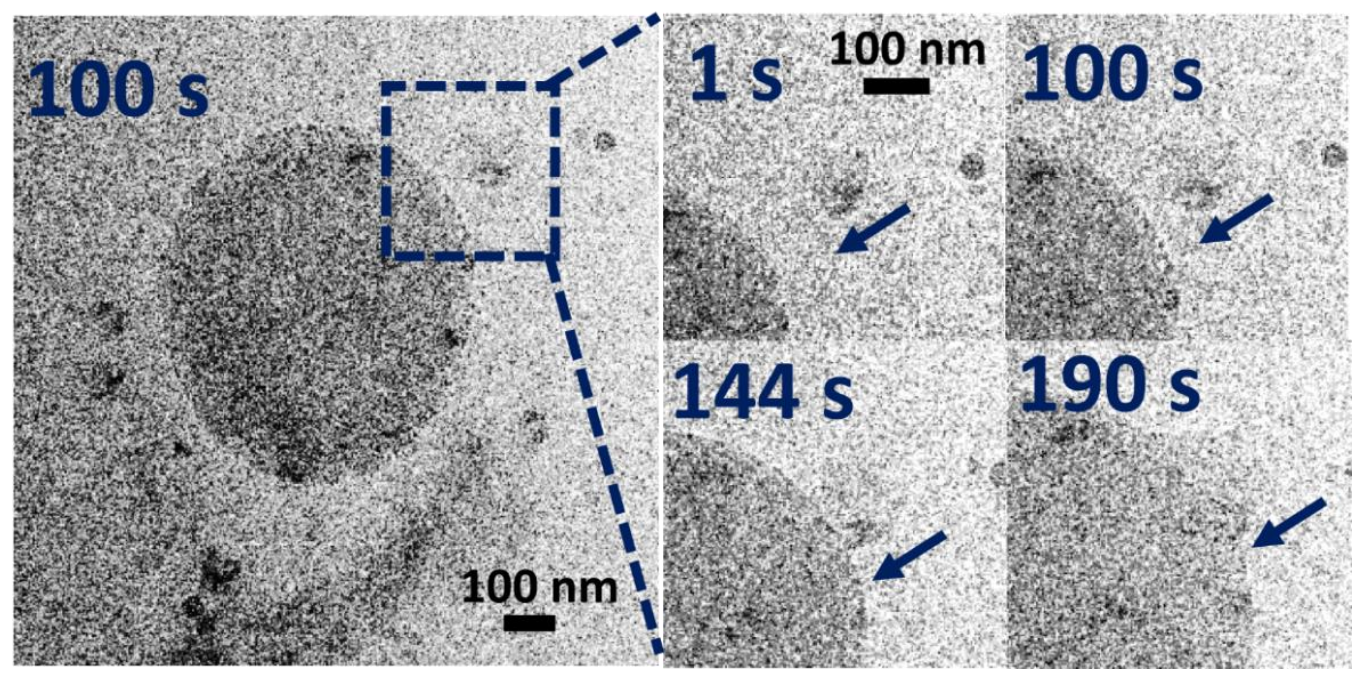

Figure 1. Snapshots from LP-TEM movie showing the growth of a polymer rich liquid droplet in the formation of PCL-b-PEO vesicles. 